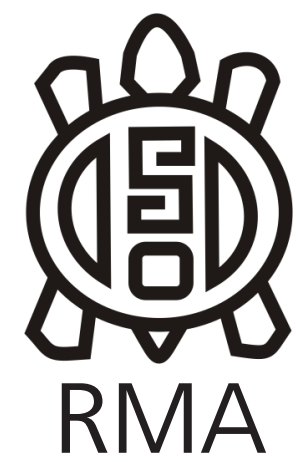

Antropología Social

\title{
Por una antropología de lucha y sangre
}

\author{
For an anthropology of struggle and blood
}

Sol Viñolo*

*Alumna del Doctorado en Ciencias Antropológicas - Universidad Nacional de Córdoba - Ayudante de Investigación en el IDACOR - CONICET / UNC.

\begin{abstract}
Resumen
En este texto Viñolo realiza un ensayo reflexivo sobre la génesis y el desarrollo de sus experiencias como etnógrafa y como militante del Partido Obrero. Se apoya en autores como Wacquant, Favret-Saada y Bourgeois para extender propuestas que han buscado radicalizar las dimensiones afectivas, sensibles, corporales de las alternativas necesarias para interrogar realidades sociculturales próximas pero esquivas para el inconsciente, distantes pero complejas para capturar el sentido del mundo para Ixs otrxs.
\end{abstract}

Palabras clave: Reflexividad; Etnografía enactiva; Partido Obrero; Afectación en el trabajo de campo

\begin{abstract}
In this text, Viñolo carries out a reflexive essay on the genesis and development of her experiences as an ethnographer and as a militant of the Partido Obrero (Workers' Party). She draws on authors such as Wacquant, Favret-Saada and Bourgeois to extend proposals that have sought to radicalise the affective, sensitive, bodily dimensions of the alternatives necessary to interrogate sociocultural realities that are close but elusive to the unconscious, distant but complex in order to capture the meaning of the world for others.
\end{abstract}

Key-words: Reflexivity; Enactive ethnography; Partido Obrero (Worker's Party); Fieldwork affectation

Es evidente que el título del artículo parafrasea aquel texto de Wacquant (2019), "Por una sociología de carne y sangre", posterior a su investigación en un club de boxeo en un barrio bajo de Chicago. En breves páginas, el autor desarrolla allí la propuesta y la apuesta metodológica de una "etnografía enactiva", que consistiría en la inmersión profunda de le etnógrafe en la estructura social y simbólica de la comunidad que nos proponemos estudiar. Esta marca del trabajo de campo inmersivo, basado en "realizar el fenómeno", es, según el autor, un camino fructífero hacia la captura de los habitus que generan las prácticas y subyacen en el cosmos que se está investigando. Contra la visión dualista y desencarnada, que nos dejan con una comprensión incompleta e inadecuada de lo social, Wacquant propone una sociología "de carne", refiriéndose a la centralidad del cuerpo vivo en la producción de conocimiento y "de sangre", apuntando "al circuito interno de la vida que pulsa en lo profundo del cuerpo visceral". Postulando una "sociología carnal" que procure comprender la "acciónen-su-construcción" y que mediante ella pueda producir información multidimensional capaz de captar la vida tal como se desarrolla en realidad.
Sí en el título de este artículo he optado por conservar el término "sangre" y no el de "carne", esto ha sido más en pos de un juego léxico que de una decisión teórica o metodológico particular, pero también para enfatizar en esta noción de "sangre" que nos propone Wacquant y en la vitalidad que implicó en mi caso, aceptando implícitamente el rol del cuerpo como su condición de posibilidad. Asimismo, me refiero a la antropología en lugar de la sociología ya que es ella mi disciplina de formación y mi campo de acción; y digo de "lucha y de sangre", para referirme a mi experiencia etnográfica en una organización trotskista argentina en la que realicé una inmersión tan profunda que llegué a convertirme en una nativa plena, sujeto de derechos y obligaciones partidarias. Sin embargo, el "hacerme militante" no fue algo premeditado como una suerte de iniciativa metodológica de investigación para conocer desde lo más adentro posible los procesos sociales que quería investigar, sino que fue el resultado del camino al que me llevó una particular sensibilidad etnográfica y el campo mismo.

Mi propia trayectoria personal y mi posición social, por decirlo de algún modo, tuvieron mucho que ver en el 
derrotero de esta transición de etnógrafa a militante. En primer lugar, resulta insoslayable el hecho de que nací en una familia "de izquierdas". Mi abuela materna había sido una activista en su juventud, presa política en el Cordobazo y exiliada en el '74 después de que la triple A asesinara al hermano de mi abuelo. Por su parte, mi mamá y mi papá se conocieron de muy jóvenes militando en el Partido Obrero y yo, que fui su primera y única hija en común, crecí en locales partidarios, entre reuniones y marchas. Mis primeros recuerdos se confunden con las historias que me contaron y con las imágenes de las pocas fotos que tenemos de esos tiempos, pero hay algo en la "sangre", en lo profundo del cuerpo visceral, inenarrable, que me recorre por el cuerpo, me acelera los latidos y, a veces, me empaña la mirada cuando pienso en los primeros años de mi infancia. Mi papá siempre cuenta una anécdota (generalmente los días de mi cumpleaños, cuando se reencuentran mis dos familias, separadas poco tiempo después de que cumplí un año), de una vez que lo llamaron desde la sala de tres a la que iba para decirle que estaba organizando a mis compañeritxs en una huelga. No tengo ningún recuerdo de eso, pero era evidente que algo de la militancia de mis xadres me "afectaba".

Por diferentes motivos, mis viejxs dejaron de militar antes de que pueda hacer más "consciente" o poder ponerle palabras a esa experiencia vivida. Mi mamá trabajaba de moza de noche y durante el día se repartia las horas para dormir, cuidarme y estudiar una carrera universitaria que le pudiera abrir nuevos horizontes; y la militancia le requería demasiado tiempo, así que se alejó del partido. Más o menos lo mismo le ocurrió a mi papá, aunque también fueron algunas discusiones políticas hacia dentro del partido las que les llevaron a alejarse en uno y otro caso (muchos años después sabría que cuanto más diferencias tenés con la dirección partidaria, más tiempo tenés que dedicarle a militar para poder defender tus ideas; $y$, justamente, el tiempo era algo que les apremiaba en ese entonces). Mi mamá no me hablaba mucho de su militancia en el PO ni de política en general. Después de algunas pocas charlas que en su mayoría inicié, comprendí que ella estaba enojada con el partido y que le había decepcionado profundamente que la organización a la que le había dedicado muchísima energía desde sus 15 años, le hubiera dado la espalda cuando "estaba pasando hambre" como ella me decía cuando le preguntaba, generándome una profunda tristeza. Mi papá, en cambio, siempre decía que él quería volver a militar cuando la vida se lo permitiera y me contaba orgulloso que él había sido un militante apasionado.

Sí bien mis viejxs nunca me "adoctrinaron", por decirlo de alguna forma, y desde que tengo memoria ya no militaban, una biblioteca abundante de literatura marxista despertó mi interés desde muy chica y las largas conversaciones con mi papá cuando le preguntaba qué significaban los conceptos que titulaban aquellas obras, me llevaron también a querer "formarme" en política.
Recuerdo un día que viajábamos juntxs en el 147, cuando le pregunté qué era el marxismo. Yo tenía ocho años y él estaba feliz de que su hijita le hiciera esa pregunta. Entonces me preguntó: "sí vos cocinás una sopa, leés en una receta cuánta sal hay que ponerle o la vas probando para ver cómo está?". Y yo, que nunca había cocinado, le dije que la probaría. Me tendió la mano y me dijo "ya sos marxista". No sé cuánto tiempo habrá pasado hasta que entendiera porqué había hecho esa analogía. Unos años después, un día le pedí prestados tres libros suyos, tras repasar lentamente todos los tomos de su estantería. Elegí: "Las formas del trabajo y la historia" de Pablo Rieznik; "Teoría marxista y estrategia política" de Jorge Altamira y "La revolución traicionada" de Trotsky. Recuerdo el asombro y el orgullo de mi papá por la elección de los libros que había hecho, pero yo no tenía mucha idea. En ese entonces estaba por cumplir 14 años y seguí leyendo teoría política buena parte de mi adolescencia, junto a otros libros, de literatura rusa, sobre todo.

Por motu propio, siempre participé de los centros de estudiantes de las escuelas por las que transcurrí, en centros culturales y todo tipo de lucha desde la primaria hasta la universidad. Pero mi militancia la había hecho sobre todo en el guevarismo, con muchas críticas al PO y a la izquierda partidaria en general. Cuando cursaba el cuarto año de mi carrera de grado en antropología, una sensación difícil de poner en palabras me llevó a interesarme en la literatura como posible objeto de estudio. Era el 2014, una tarde de domingo después de ver la película homónima de Anna Karenina de Tolstoi, que me hizo revivir aquellos años en los que me devoraba los clásicos rusos. Fue una especie de epifanía que me hizo saltar de la cama, sabiendo que estaba frente a la magia en la que me quería sumergir, tanto para entenderla como para poder decir de ella su poder transformador. Supe así, mediante una manifestación instantánea, que allí estaba mi objeto de tesis. De los rusos a la formalización de un proyecto de tesina de grado, fue un largo derrotero. Acoger lo básico de la investigación formal académica, implicó un recorte brusco y un giro metodológico fundamental para encajar mis enormes pretensiones en una tesis asible. El tema que delineé, no sin vueltas, fue la literatura en el ámbito de la política. Al haber llegado allí persiguiendo la curiosidad por esa magia de la literatura de ficción, capaz de crear mundos, supe desde un primer momento, que había hallado mi lugar en los márgenes e intersecciones de las disciplinas. Así, entre las letras, la filosofía y la antropología, y próxima a los estudios sobre el libro y la edición, terminé por darle forma a un trabajo final de licenciatura, que tenía como hipótesis en el horizonte un talismán que me acompaña todavía, como si en realidad ese fuera el verdadero hilo conductor de mis búsquedas, más aún que el multiverso de la escritura, que era a la vez una pregunta: ¿puede la antropología llenar esa distancia abrumadora que escinde las disciplinas, entre el texto y el mundo de las prácticas 


\section{humanas?}

La elección de hacer el trabajo de campo en dos partidos políticos se debió a que quería que la política constituyera una parte significativa de las actividades de las personas con las que quería trabajar y que ciertos marcos de copertenencia marcasen algún grado de trayectoria común entre ellas. Motivada por mi mentor, Gustavo Sorá, opté por hacer un gran contraste en vez de concentrarme en la izquierda como me había imaginado en un primer momento, y elegí así un partido de la derecha y uno de la izquierda. Opté por la UCR por ser uno de los más antiguos del país y porque no quería meterme en el eclecticismo del peronismo. De la izquierda dudé entre el PC, el PTS y el PO, ya que todos tenían una producción escrita prolífica, hasta que finalmente me decidí por el PO. Sí bien me asustaba ser "marcada" por parte de lxs militantes más antiguos que conocían a mis xadres, me favorecía el hecho de tener algunos contactos lejanos de los cuales valerme para ingresar al campo; y esto último fue lo que me llevó a decidirme. En retrospectiva, siento que algo de la historia de mi familia y mi necesidad por entender esas pasiones que hasta a mi misma me había impulsado en mis primeros años de vida, tuvieron también mucho que ver en esa elección, aunque en ese momento no fuera consciente de ello.

Mi primer acceso al campo fue a través de una entrevista con Eduardo Salas, que conseguí a través de estos contactos. En realidad, no fue tanto la entrevista en sí, sino que en ese encuentro Eduardo, "el largo" como yo lo conocí, me contó que en menos de dos semanas después se iba a hacer una presentación de En Defensa del Marxismo, la revista teórica del PO, a cargo nada más y nada menos que de Jorge Altamira, a quien le guardé siempre una profunda admiración transmitida por mi padre. Era una oportunidad que no podía desaprovechar

Hacía tres semanas que había comenzado el trabajo de campo en la Unión Cívica Radical, el laberinto de ausencias de cultura escrita con el que me tropecé desde la primera vez que entré en la Casa Radical (como investigadora y en todo concepto), iban perfilando un trabajo que se asemejaba más a la historial oral que a una etnografía de participación y diálogo. El contraste entre ambas comunidades partidarias fue crucial, en ese sentido, para establecer una relación fundada en el devenir histórico de cada partido: mientras que en la UCR, la pluma en el escudo había dado paso a la ausencia casi absoluta de presencia y circulación de la escritura en el presente; en el Partido Obrero la cultura escrita ocupaba un lugar fundamental en el desarrollo político de de la organización. Pero no me detendré en eso aquí.

La jornada que se convertiría en mi primer día de trabajo de campo en el PO, estaba convocada para el día jueves 30 de octubre de 2014 a las 19:30 hs, en el Aula 1 del Pabellón Haití de la Facultad de Artes, en ciudad universitaria. Me presenté puntual (con la sospecha del carácter impuntual de la organización que efectivamente más adelante corroboré) y la actividad no sólo no estaba por empezar, sino que recién comenzaban a preparar el aula, mientras la gente iba llegando de a poco; ese margen me dio tiempo de acercarme y, por fin, romper el hielo, lo que me resultó casi espontáneo y definitivamente gratificante. Lo primero que vi... ahí estaban, como esperándome, los folletos de todos los colores, el periódico, ¡los libros!, y las insignes banderas amarillas y rojas. Se trataba de una mesa que por supuesto me apresuré en fotografiar, después de observar detenidamente cada una de las cosas que en ella se encontraban. Claro que el hecho de que una persona (ajena) le saque fotos a una mesa de materiales no pudo menos que atraer algunas miradas, las que, en todo caso, me sirvieron de puente y oportunidad para presentarme y contar por qué esa mesa me producía semejante complacencia. Así que ya estaba ahí, ya había dicho quién era y qué hacía por lo menos a alguien, y lo que siguió fue una seguidilla de repetición del brevísimo discurso a unas cuatro personas a las que me fueron derivando ("podés hablar con tal..."), hasta que se hizo la hora de ingresar al aula para dar comienzo a la charla. Para ese momento ya tenía una agenda de nombres a quién visitar, y, más importante aún, ya me había comprometido a viajar al Congreso del Movimiento Obrero y la Izquierda, que se realizaría el 8 de noviembre en el Luna Park, Buenos Aires, al que me invitó un militante de PO, escritor y profesor de literatura.

Al finalizar la charla pude hacerle una pequeña entrevista a Jorge Altamira sobre la función de la cultura escrita en el PO y la relación de la literatura y la política para él. Y después entablé relación con Irene, estudiante de bibliotecología, militante del Partido Obrero y encargada de restaurar la biblioteca del partido en el nuevo local partidario, ubicado en Mariano Moreno 69. Inmediatamente me ofrecí para ayudarle en lo que hiciera falta, ya que mientras le ayudaba podría aprovechar para conversar en profundidad con ella y "estar ahí" en el partido y en el local; le pasé mis horarios de trabajo y quedé a su disposición. Era el primer día de trabajo de campo en el PO y había dado dos pasos fundamentales. En ese momento sólo lo intuía, pero tiempo después pude llegar a la conclusión de que el viaje al Congreso y el trabajo en la biblioteca fueron dos claves en el desarrollo de mi investigación, en ambos casos por la red de relaciones que me propiciaron. Al llegar a mi casa esa noche tenía cuatro libros que me había comprado, la prensa, un compromiso de viaje, al menos siete contactos en mi teléfono, una hora de grabación de la charla, una entrevista hecha, fotos y una agenda de trabajo.

Tal como acordamos esa primera noche en la presentación de EDM, Irene se comunicó conmigo a los pocos días, dispusimos un horario fijo y empezamos a trabajar juntas en la biblioteca. El primer día que quedé con Irene, me 
presenté en el local, saludé, pasé y me instalé con ella en el pequeño espacio que hacía de recepción y que contenía las estanterías con los libros, ubicado al lado de la única puerta de ingreso al local. Irene, que sabía mucho más de cómo armar una biblioteca que yo, disponía cómo hacer el trabajo. Primero recortamos unos papeles y los pegamos en el lado interior de la contratapa de algunos libros; después hicimos un inventario y acomodamos un poco. Mientras, yo aprovechaba a hacer preguntas sobre todo lo que podía.

A pesar de los nervios y la timidez que siempre me caracterizó, sentía una extraña sensación de placer. Muchxs militantes que salían de sus reuniones pasaban por esa pequeña sala, me saludaban, me miraban con inquietud, pero no era eso lo que me generaba placer ni siquiera el hecho de estar "cumpliendo" con mi objetivo de hacer trabajo de campo, sino otra cosa, una sensación para la que nunca tuve palabras, que me la provocaba el hecho mismo de estar en el local. Sensación que siempre sentí en los años posteriores. A veces llegaba a pasar días enteros, incluso hubo algunas noches excepcionales que dormí en el local y siempre tuve la misma sensación, que era similar pero potenciada a la sensación de tener las llaves de allí, lo cual ocurrió mucho tiempo después.

Las innumerables discusiones políticas que tenía con militantes del partido hicieron de alguna forma que me sintiera cada vez más cercana a la organización. La predisposición a discutir por parte de Ixs militantes del PO, en este sentido, tuvo mucho que ver con que yo pudiera deshacer algunos prejuicios y comprender el accionar político del partido. Asimismo, ese afán de invitarme e insistir en que participe de las actividades a las que nunca me rehusaba, me llevaron a participar de reuniones de círculo, congresos y viajes partidarios cada vez con más frecuencia. Primero como etnógrafa, después como invitada cercana, luego como medianamente militante, aunque todavía hacía mi tesis, registraba e incluso hacía entrevistas a algunas personas; pero mi presencia ya no era extraña. Y así fue que, en un momento, que no puedo determinar a ciencia cierta cuándo fue, ellxs, mis interlocutores de campo, borraron primero esa distancia conmigo y después yo misma me asumí dentro de la organización.

Por supuesto que convertirse en miembro activo llevó tiempo, pero no tanto en realidad. La intención de sumergirme cada vez más en la corriente de acción a la mayor profundidad posible - "en lugar de mirarlo todo desde la playa" (Wacquant)- se conjugó con esa actitud sedienta del partido por absorber siempre a más personas nuevas y asignarle cada vez mayores responsabilidades. Tras tres meses de trabajo de campo, ya participaba de reuniones de círculo donde tenía acceso al calor de las discusiones partidarias; y dos meses después participé por primera vez de un congreso provincial que se realizó a medidados de enero de 2015 en "La morocha", un salón alquilado que a la noche funcionaba de boliche, ubicado en pleno centro cordobés, con el interior todo pintado de negro, donde pasé uno de los días más calurosos de los que me acuerdo.

En abril de ese año me "recomendaron" que pase del círculo de Río Ceballos donde había comenzado a participar porque en ese entonces vivía en la localidad vecina de Unquillo, a "militar" en la juventud. De algún modo Ixs militantes me habían asignado ese rol y me parecía bien aceptar la propuesta. En ese entonces yo hacía tiempo que no pisaba la facultad, hacía un año que había dejado de cursar materias de antropología y que no transcurría más por los pasillos de la facu y por ciudad universitaria en general.

Fue así que una tarde de abril, dos compañerxs con quienes había hablado únicamente por teléfono me esperaron en la "mesita" de la facu de filo para sumarme a las actividades de la juventud. Una mesita pintada de rojo que pocas veces había llamado mi atención en los años de carrera y que ahora pasaría a tener un sentido completamente nuevo. Ese día fuimos a la facu de psico y nos pusimos a pintar carteles, que era una de las actividades más asiduas y que comúnmente se le asignaban a compañeres que recién se acercaban a la militancia. Después de unas cuantas horas de estar allí, se hizo de noche y las personas que nos encontrábamos en psico y otras que llegaron desde otras facultades nos reunimos improvisadamente cerca del comedor universitario. En esa reunión me hicieron una especie de bienvenida.

Después de ese día, no sé con qué vertiginosidad se precipitaron los acontecimientos que siguieron, pero hasta me mudé a Córdoba. La agenda de la juventud era intensa. Todos los días había una u otra actividad, y si estabas disponibles allí tenías que estar. En mi caso, que trabaja solamente durante las mañanas como secretaria en un estudio jurídico, tenía el resto de la tarde y hasta la noche para las actividades de militancia que no sólo implicaban estar en la unversidad, poner la mesa, hacer carteles, pasar por los cursos, etc; sino también estar en las calles, en las concentraciones y marchas que al menos se realizaban una vez por semana. Muchas veces después de las movilizaciones había que llevar las cosas al local (banderas, pancartas y tambores, entre otras) y casi siempre la juventud estaba encargada de ello. Volver caminando al local cargando las cosas por las diez o quince cuadras que había generalmente desde algún punto en el centro hasta el domicilio del local en el barrio de alberdi, con el cansancio de haber estado agitando por horas en la manifestación, era otro de los momentos que de alguna forma misteriosa me provocaban mucho placer. Una vez que llegábamos al local, generalmente nos quedábamos tomando las cervezas más baratas y cenando maní y papas fritas que comprábamos con los pocos pesos que juntábamos entre todes, mientras hacíamos 
una reunión de balance de la actividad o simplemente nos relajábamos escuchando las Manos de Filippi, hasta terminar coreando "organización internacional...". Puedo decir que allí y entre todo ese trajín de la militancia en la juventud hice verdaderas amistades, quizás algunas de las más profundas e importantes que tuve en mi vida y que conservo todavía. Compartir la actividad cotidiana de la militancia, me llevó a generar un tipo de afecto que desconocía hasta entonces, a pesar de haber militado muchos años de mi vida en otro tipo de organizaciones, como en las agrupaciones estudiantiles y barriales guevaristas donde había generado vínculos de amistad; había una diferencia muy grande entre lo que se podía llegar a compartir en los encuentros semanales que teníamos y alguna que otra "reunión social" con estos grupos, en relación a la militancia cotidiana, del día a día, de la mañana a la noche, que implicaba militar en el Partido Obrero, sumado a los enormes objetivos y las convicciones políticas en común.

El partido en su conjunto se reúne en sus respectivos nucleamientos después de que sale el nuevo número del periódico, ya que es a partir de la lectura de su editorial que se debate la "situación política". Como el nuevo número sale siempre los días jueves, la dirección regional se reúne lo antes posible, generalmente el mismo jueves a la noche, esos debates y definiciones de acción se discuten después en los comités y los subcomités y después en los círculos. Eso hace que el sábado sea siempre el día predilecto para que se reúnan todas las agrupaciones y los círculos de partido, además de las ventajas que tiene que sea un fin de semana, para que puedan asistir la mayor cantidad de compañerxs. En general, los sábados eran siempre los días más cargados. El movimiento en el local esos días era constante, la gente entraba y salía permanentemente. Por momentos, se quedaba en silencio, cuando cada grupo estaba reunido en una sala diferente, pero de pronto alguna de las reuniones terminaba y se podían escuchar las voces de les militantes que salían y los pasos crujientes de los pisos de madera de la pequeña casona antigua. Los días sábado se realiza también una "agitación" en la peatonal, en pleno centro cordobés, que consiste en llevar una mesa y algunas banderas y entre cuatro, cinco o seis compañerxs que van ofrecen el periódico y reparten volantes buscando entablar alguna discusión y sacar contactos. La juventud siempre estaba indicada para participar de esa actividad y así lo hacíamos en muchas oportunidades. Esos días eran especialmente largos. Había que madrugar para estar a las 8 en el local para buscar las cosas y trasladarlas caminando hasta el punto que generalmente era en la esquina del cabildo en la plaza San Martín o en la esquina de la legislatura, a una cuadra de distancia la una de la otra. Llevar las cosas hasta allí era bastante engorroso; la mesa plástica era particularmente molesta. Más complicado era todavía cuando había que llevar el parlante para poner los spots publicitarios en épocas de campaña electoral, para que la agitación sea más vistosa y atractiva. Las banderas también tenían su complejidad ya que por el largo de los palos había que llevarla entre dos personas, cargadas en los hombros, pero siempre disfruté de llevarlas, incluso cuando el bulto era tan grande que el peso hacía doler la espalda, pero eso era en las marchas. Una vez que llegábamos a la peatonal, desplegábamos rápidamente las banderas y pancartas pegándolas a los postes con mucha cinta de embalar, mientras otres disponían los periódicos y algún otro material impreso de alguna campaña específica en las mesas. Y a los pocos minutos ya estábamos todes ofreciendo el periódico simultáneamente, pero entre una cosa y la otra casi nunca arrancábamos antes de las 10 . El sol del mediodía rajaba y a falta de alguna sombra nos refugiábamos en las gorras rojas y amarillas del partido y en los lentes de sol que muchas veces a varies nos ayudaban también a disimular alguna resaca. Lo máximo que llegué a vender en una de esas mañanas fueron 23 periódicos. Cuando se acercaba la hora de comer y los locales comerciales comenzaban a cerrar, el movimiento de transeúntes disminuía. Esto ocurría, generalmente, entre las 13 y las 13:30 hs, cuando "levantábamos campamento" y regresábamos al local, cansadxs y hambrientxs. Hubo muchas veces, que después de la agitación en la peatonal, nos cocinábamos en la cocina del local y comíamos antes o durante alguna reunión que hacíamos en la misma cocina, la cual no era muy grande pero una importante mesa ovalada de madera ubicada en el centro nos brindaba suficiente comodidad para comer y desplegar periódicos y cuadernos donde garabateábamos una que otra anotación. En general el menú de esos almuerzos era fideos con crema, arroz blanco con arvejas o sandwiches de pan francés, mayonesa, queso y mortadela. Los utensilios eran mínimos: una única olla de lata, un jarrito, un puñado de platos de diferentes colores y tamaños y unos pocos cubiertos donados que nos turnábamos para poder comer. Después de que varias veces me tocara abrir latas con el único cuchillo que había, doné un abrelatas que tenía repetido en mi casa. Al resto del partido (más "adulto") no le hacía mucha gracia que la juventud haga semejante despliegue en la cocina, pero lo hacíamos igual. Ese era el espacio por el que todes pasaban en algún momento de su estadía en el local partidario, generalmente a poner o sacar agua de la pava para el mate; y nos hacían algún que otro comentario al respecto. En general, las reuniones se interrumpían en muy pocos casos, pero cargar el termo y arreglar el mate era uno de ellos. Las horas pasaban y seguíamos en el local, a veces se hacía de noche antes de irnos. En los ratos que no estábamos en reunión se aprovechaba el tiempo para hacer otras cosas, como por ejemplo editar algún flyer, volante o boletín y después imprimirlo. Así fue que aprendí a usar el corel en la única computadora del local que se encontraba en la salita de recepción y que funcionaba con una especial lentitud capaz de exacerbar la paciencia de cualquiera. Las fotoduplicadoras que se encontraban en un pequeño cuarto arriba de la cocina al que se subia por una destartalada escalera caracol de chapa, también 
tenían lo suyo; a veces se trababan o imprimían mal, pero dentro de todo, había aprendido a dominarlas.

El 2015 fue un año de mucha actividad militante. Cada vez participaba de más actividades y asumía mayores responsabilidades. Estar en la juventud implicó un salto significativo en ese sentido. Como el círculo de "filo" no estaba conformado y ese era mi espacio "natural" de militancia, como me decían, ya que se trataba de la facultad en la que yo realizaba mis estudios; me asignaron la tarea de armar ese círculo, dirigirlo y por lo tanto participar de la dirección de la juventud de capital. El desafío que implicaron estas tareas sería objeto de un artículo extra. Para mí significó meterme de lleno a la actividad militante, mientras continuaba con mi tesis. Los días domingo que en general eran de mucha menos actividad partidaria, los dedicaba a desgrabar y escribir.

Mi investigación, que era sobre el lugar de la literatura y la cultura escrita en el partido, quizás podría haber sido pasible de indagar sin semejante inmersión, pero dudo que pudiera haber producido un conocimiento tan profundo. No hay que tener demasiadas cualidades para observar el rol protagónico del periódioco partidario en el PO, casi siempre Ixs militantes tienen periódicos en sus mochilas, se venden en todas las actividades y lo más probable es que si te cruzás con un militante del PO quiera venderte un Prensa Obrera. Ahora, solo quien haya intentado vender el periódico y lo haya hecho más de una vez, puede saber, por ejemplo, de la real eficacia de conocer el contenido de cada número y más aún mientra más lo ha leído, para poder vender ese periódico. Fuera de los pocos casos de la gente que en una marcha te compra la prensa por "colaborar", en general, efectivizar la venta de la prensa depende de la discusión que puedas desenvolver sobre los temas que desarrolla el periódico, transmitiendo la necesidad de leerlo para comprender la realidad política coyuntural general o algún tema particular. Necesidad que, a su vez, cada militante siente efectivamente, ya que es a través de la lectura del periódico que cada militante se empapa de los principales debates políticos nacionales. Este caso es un ejemplo de los datos precisos a los que se puede acceder a través de una inmersión profunda en las actividades de nuestros interlocutores, "realizando el fenómeno" que queremos investigar. Otro ejemplo podría ser la pregunta por las motivaciones que llevan a Ixs militantes a dedicar tanto tiempo de su vida a la militancia. Alguien externo podría pensar que lo hacen por prestigio, por reconocimiento, por poder incluso, y no es que no haya nada de esto en absoluto, pero las motivaciones son otras y sólo son accesibles a través del estar ahí y de inmiscuirse en la actividad militante. Estas motivaciones, además, no son verbalizables, o solo una parte de ellas puede ser dicha, pero el efecto que te lleva a levantarte a las 5 de la mañana en pleno invierno para ir a cortar un puente, no es solamente la convicción por la reivindicación por la que se lucha en ese momento, por más legítima, urgente y necesaria que sea, y no es posible de capturar o aprehender sin estar ahí, en el medio de los cánticos, riendo con tus compañerxs por lo desafinado que suenan los instrumentos, cuando asoma el sol y la gente te grita desde los autos que vayas a laburar sin saber que lo más probable es que estés mirando a cada rato el reloj para saber cuando tenés que irte para llegar a tiempo a tu trabajo, o cuando se aproxima la policía y los cantos se hacen más fuertes para adelantarse a cualquier violencia institucional.

\section{Dejarse afectar}

El dispositivo metodológico que propone Favret-Saada, que se trata del "ser afectado", como el impacto que ocasiona una experiencia bajo una forma inconsciente o energética que sólo es aprehensible para le etnógrafe a través de su experimentación directa, dejandose afectar por las lógicas de funcionamiento de los fenómenos que se quiere analizar, es propicio para analizar mi propia experiencia en el PO.

Este dispositivo que la autora lo implementa para estudiar los actos de embrujamiento/desembrujamiento en el Bocage francés, Favret-Saada lo desarrolló para lograr comunicarse con los habitantes del Bocage, ya que por un lado, éstos interponían una barrera de mutismo en relación al tema, y que por el otro, buena parte de esa comunicación no se realizaba a través de palabras ni de las acciones emprendidas; sino que era algo no representado, aprehensible únicamente a través de la transmisión de una carga energética que era leída en el comportamiento y la apariencia de los interlocutores. Es por ello que, según la autora, es fundamental para la Antropología Social y para el trabajo de campo etnográfico, no sólo el "estar ahí", siendo esa presencia el requisito mínimo para que la observación sea posible, sino "dejarnos afectar por las realidades que viven y experimentan nuestros informantes e interlocutores, abandonando nuestro principio de orientación etnocéntrico como única medida de la realidad y de las teorías que elaboramos".

En este sentido, Favret-Saada, propone también reconsiderar la noción de afecto y reparar en la importancia de la exploración de su significado, para rehabilitar la antigua noción de "sensibilidad", poniendo en cuestión la limitación de la antropología al estudio de los aspectos intelectuales de la experiencia humana. Asimismo, afirma que ocupar un lugar en el sistema de la brujería, en su caso, no le informa nada sobre los afectos de les otres, pero que por "el solo hecho de aceptar ocupar esa posición y ser afectado por ella abre un tipo de comunicación específica con los nativos: una comunicación de todo involuntaria y desprovista de intencionalidad, que puede o no ser verbal".

En mi caso, lejos de sostener un mutismo, Ixs militantes del PO se apasionaban por contar de su militancia, del partido y de cualquier cosa al respecto que les pudiera 
preguntar. Y sin embargo, habían un montón de otros aspectos no verbalizables de los cuales pude dar cuenta a través de dejarme afectar por la militancia trotskista y hacer una inmersión profunda en el hábitus militante piquetero, fundamentalmente a través del afecto y la sensibilidad.

Cuán cierta fue para mí la propuesta metodológica de Favret-Saada. Y no lo fue sólo por ocupar un rol social en el campo, ni por los afectos y las amistades que entablé. La militancia en el PO me afectó decisivamente, alteró mis rutinas, mis círculos afectivos, mis intereses profundos, hasta mis gustos musicales e, incluso, estar en la juventud me "rejuveneció", siempre lo sentí así. Eran muches de mi misma edad e incluso más grandes, que militábamos en las facultades. El ritmo de militancia, las personas con las que discutíamos y las actividades que hacíamos, todo me llevaba cada vez a sentirme parte de esa "juventud"; también porque ese era el lugar que teníamos les jóvenes en el partido y porque poco a poco empezé a sentirme cómoda allí.

Militar en el partido también me dejó otras enseñanzas. Me empujó a hablar en las asambleas de lucha y en los congresos partidarios, algo a lo que siempre me había negado, pero la necesidad de hacer un planteo particular que "tenía que hacer", me llevó a superar los nervios de a poco y romper el silencio. También me propició oportunidades para desarrollar nuevas habilidades, desde flamear una bandera hasta organizar grandes eventos.

En los años de trabajo de campo y en los que siguieron de militancia una vez finalizada la tesis, me dejé afectar profundamente, al punto que me es imposible todavía escribir y que no se me humedezcan los ojos añorando aquellos tiempos. El vacío que sentí después que dejé de militar también me sirvió para reflexionar, "entender" desde la vivencia del dolor, del sufrimiento, de la nostalgia visceral, la enorme demanda de energía que implicaba militar en el partido, una demanda imposible de satisfacer completamente y que alcanzaba y sobraba para llenar la vida de cualquiera o al menos la mía. Pero no se trataba de "llenar" las horas del día con actividades, sean cuales fueran, sino de llenar la vida emocional y pulsionalmente, llenarla de adrenalina y de convicciones políticas viscerales, que te atraviesan entera, desde la formulación en palabras de un programa anticapitalista, clasista e internacional, hasta la fuerza que sale desde las entreñas o de no sé donde y que se incrementa en el pecho para cantar con una voz mucho más fuerte y grave de lo normal, para denunciar una injusticia, un genocidio o una única muerte, como la de Mariano, que se lleva los cantos más fuertes más piel de gallina, seguido por los cánticos que reivindican los métodos piqueteros. $Y$ es que hay un sentido de pertenencia tan fuerte, tan arraigado, que se enuncia como ser "piqueterx" y se manifiesta en la lucha, en la reivindicación del método del corte de ruta, que rememora el Argentinazo y todas las luchas contra las opresiones sociales, que se "opone por el vértice" a todas las políticas de los regímenes gobernantes y sus aliadxs. No sé cuán profundas hayan sido las experiencias de embrujamiento/desembrujamiento en el Bocage que llamaron la atención de Favret-Saada por cuarenta años; sólo puedo decir y atestiguar con mis vivencias, que la energía y la vitalidad de la militancia en el PO que en mi caso se potencian en algún lugar del inconsciente con la conmoción generada en mis primeros años de vida y el afecto profundo por mis xadres, es una de las experiencias más vigorosas por las que he atravesado en toda mi vida. Sí me llevó ocho años escribir sobre esto, fue porque yo misma había caído en el prejuicio positivista que Bourgois ${ }^{1}$ nos anima a romper para reunir datos precisos en etnografía, es decir, datos intersubjetivos que dependen de las relaciones personales de confianza y de afecto con Ixs otrxs; siendo justamente la experiencia vivida por le etnógrafe en relación con les otres nuestro medio de conocimiento para estudiar los procesos sociales de nuestro interés, que en mi caso era la cultura escrita y la literatura en el ámbito partidario.

Sí Wacquant dudó en quedarse en el club de boxeo y abandonar la investigación sociológica a la que se dedicaba, esta nunca fue una cosa que yo me cuestionase. Mi carrera académica continuaba y mi militancia, indudablemente, también; y así lo fue durante algunos años.

No fueron pocas las diferencias políticas que me alejaron del PO en un momento y que después hicieron que me echaran, junto a otrxs más de mil compañerxs de todo el país, cuando me pronuncié en favor de la tendencia interna que se conformó en el 2019 y que al ser desconocida como tal se reagrupó por fuera del partido. Pero todo esto no viene al caso. Los años de militancia en el PO fueron de los más memorables y me permitieron reunir datos precisos sobre la función neurálgica de la cultura escrita en la construcción partidaria. Deshacer las pretensiones objetivistas del positivismo académico, tal como nos proponen Wacquant, Favret-Saasa y Bourgois, entre muchos otrxs autores, cada cual a partir de sus propias experiencias de campo, es el primer paso para hacer una etnografía sensible capaz de reponer las vivencias de los procesos que nos interesa conocer, dejándonos afectar por sus lógicas, ocupando roles en su seno y sabiendo, después, registrar esa experiencia a fin de comprenderla y transformarla en un objeto de estudio de nuestras ciencias antropológicas.

\section{Bibliografía}

Favret-Saada, Jeanne. (2013). Ser afectado como método de conocimiento en el trabajo de campo antropológico. Avá. Revista de Antropología 23: 49-67.

\footnotetext{
1 N. Ed.: Se refiere al antropólogo Philippe Bourgois (1956) de la Universidad de California, muy reconocido a partir de trabajos como En busca de respeto: vendiendo crack en El Barrio (2003). No hay claro indicio de que referencia pudo haber consultado Viñolo y por eso no la incluimos en la bibliografía.
} 
Wacquant, Loic. (2019). Por una sociología de carne y sangre. Revista del Museo de Antropología 12 (1):
117-124. DOI: https://doi.org/10.31048/1852.4826.v12. $\mathrm{n} 1.24166$ 